\title{
Towards searching as a learning process: A review of current perspectives and future directions
}

Journal of Information Science

2016, Vol. 42(I) 19-34

(c) The Author(s) 2016

Reprints and permissions:

sagepub.co.uk/journalsPermissions.nav

DOI: $10.1177 / 016555151561584 \mid$

jis.sagepub.com

Soo Young Rieh

School of Information, University of Michigan, USA

\section{Kevyn Collins-Thompson}

School of Information, University of Michigan, USA

\author{
Preben Hansen \\ Department of Computer and Systems Sciences, Stockholm University, Sweden
}

\author{
Hye-Jung Lee \\ Institute for Education and Innovation, Republic of Korea
}

\begin{abstract}
We critically review literature on the association between searching and learning and contribute to the formulation of a research agenda for searching as learning. The paper begins by reviewing current literature that tends to characterize search systems as tools for learning. We then present a perspective on searching as learning that focuses on the learning that occurs during the search process, as well as search outputs and learning outcomes. The concept of 'comprehensive search' is proposed to describe iterative, reflective and integrative search sessions that facilitate critical and creative learning beyond receptive learning. We also discuss how search interaction data can provide a rich source of implicit and explicit features through which to assess search-related learning. In conclusion, we summarize opportunities and challenges for future research with respect to four agendas: developing a search system that supports sense-making and enhances learning; supporting effective user interaction for searching as learning; providing an inquirybased literacy tool within a search system; and assessing learning from online searching behaviour.
\end{abstract}

\section{Keywords}

Assessment of learning in searching; expertise modelling; searching as learning; web searching behaviour

\section{Introduction}

Reviewing the current literature on students' searching behaviour with respect to learning tasks reveals a contradictory picture. Some studies show that students struggle to find the right keywords, formulate search queries and understand search results [1-3]. When search results do not satisfy their information needs, students tend to accept those initial results uncritically, rather than re-evaluating the results and/or revising their search statements [4]. Students are also generally reluctant to look beyond the first few search results [5]. On the other hand, other studies show that students overestimate their search skills and believe that accessing, searching, using and evaluating sources of information are easy tasks that anyone can perform [6]. As a result, students tend to skim, evaluate and select online content quickly without investing much effort [7]. In general, previous studies imply that students' search behaviour and lack of search skills are

Corresponding author:

Hye-Jung Lee, Institute for Education and Innovation, Seoul, Republic of Korea.

Email: hyejung.grace.lee@gmail.com 
the main problems: if students could only improve their search skills, they would be able to search better and possibly learn more in the process.

We adopt a different approach to the problem in this paper. We focus not on issues with students' search behaviour, but instead on the ways in which information retrieval systems have been conceptualized and developed. Historically, search systems have long been characterized as a type of tool for retrieving relevant content or finding answers that satisfy people's information needs. From this perspective, research and development on search technology tends to focus on improving the effectiveness of search results to match document with search queries. In terms of the search user interface, the majority of search systems still display a ranked list of search results. This interface design signals the expectation that people may want to select relevant documents from the search results page efficiently and quickly. Indeed, users become accustomed to interacting with search systems in quick and easy ways. Typical systems may work well for certain kinds of tasks, such as look-up tasks, but not necessarily for other kinds of search tasks, such as learning, investigation and discovery, which require iteration, reflection and analysis of results by the searcher [8]. In other words, current search engines are optimized for only a certain kind of learning - acquiring factual knowledge - but are less successful at facilitating other kinds of learning, such as understanding, analysis, application or synthesis, in terms of Bloom's taxonomy [9].

The purpose of this paper is to conduct a critical review focusing on the intersection of searching and learning. This intersection is not, in itself, an entirely new research topic: what we contribute here is an attempt to bring together three disconnected research sub-areas in the field of information science: (a) investigation of students' web searching behaviour in learning environments; (b) design of information literacy programmes to improve students' search skills; and (c) development of search systems to support student learning during searching and enhance learning outcomes and experiences. We argue that research efforts across these three areas are disconnected because the relation between searching and learning has been simply assumed. Consequently, a literature review is helpful as it shows that the problem is not any lack of empirical studies demonstrating the significance of searching as learning. Rather, the problem lies in the lack of efforts to bring together and synthesize a variety of perspectives, concepts, factors and indicators that could better explain the complex relation between searching and learning.

Recently, two information retrieval workshops took place that examined this intersection of searching and learning. The participants in SWIRL (The second strategic workshop on information retrieval in Lorne) in 2012 emphasized that information retrieval systems must play a more central role in supporting learning experiences through a variety of tools for exploring information. The SWIRL participants also proposed that information retrieval systems should support and foster a larger variety of more sophisticated search strategies [10]. In 2013, several participants in the Dagstuhl seminar on evaluation methodologies in information retrieval similarly proposed a research agenda called 'from searching to learning', emphasizing the importance of conceptualizing learning as search outcomes [11]. While it is encouraging that discussions have started in such workshops, the relation between searching and learning still tends to be taken for granted and not well articulated in the current literature, with a few exceptions [12-14].

Reviewing the relationships between searching and learning is thus a critical step to establishing 'searching as learning' as a new research agenda. This is an important research problem because search systems represent a potentially powerful learning technology that can support and enhance human learning by providing access to rich, personalized online content. In this paper, we propose a new perspective on searching as a learning process that attempts to reconfigure current search systems in order to support and improve human learning. We believe that this paper will serve as a foundational review for other researchers who are interested in investigating searching and learning, and for developers and other information professionals who want to design and develop a new kind of search system that is optimized for learning tasks.

\section{Searching as a learning tool}

Most previous research on searching and learning has been set within the perspective of either 'searching to learn' or 'learning to search' [10]. Studies on searching to learn investigate students' searching behaviour in learning contexts with respect to students' research process for school assignments $[15,16]$. On the other hand, studies on learning to search examine how students' search skills could be better developed and improved [17]. In both perspectives, however, searching is conceptualized as a tool for learning. The retrieval 'tool' is often designed to help people find information needed to further their learning goals. These perspectives take a position that information is the 'basic building block for human learning' [18: 2] and that 'developing expertise in accessing, evaluating, and using information is in fact the authentic learning that modern education seeks to promote' [19:2]. 


\section{I. Construct of learning in search contexts}

In terms of the construct of learning, there have been at least four distinct ways of conceptualizing learning with respect to searching. Traditionally, information science researchers have conceptualized learning as context for searching. Kuhlthau's [15] Information Search Process Model describes information searching as a process of constructing meaning while completing a research assignment in a learning context. This model distinguishes the learning context for searching from the work context or everyday life context. For other information retrieval researchers [14, 20, 21], learning is defined as conceptual change. This means that learning changes or reinforces existing knowledge structure. Learning has been also conceptualized as the interactive intention of searching. Interactive intentions refer to subgoals that a user seeks to achieve in the process of searching [22]. For instance, people may set the following intentions: learning domain knowledge, learning information system features, learning information retrieval skills or learning database content [22]. Finally, the taxonomy of learning (i.e. remembering, understanding, applying, analysing, evaluating, and creating) designed by Bloom and his colleagues $[9,23]$ has been used to characterize different kinds of search tasks [24-26]. Recently, Arguello et al. [24] used Anderson and Krathwohl's learning tasks [9] to develop search tasks that reflect different amounts of cognition and effort required to complete the tasks. Wu et al. [25] and Kelly et al. [26] also used Anderson and Krathwhol's [9] taxonomy when they evaluated a set of search tasks in terms of levels of cognitive complexity across multiple domains. More research is needed to better understand the construct of learning in search contexts. For the time being, it is important for researchers to define the construct of learning in each study, as learning can be defined in a variety of different ways.

\subsection{Searching to learn}

This perspective starts with the notion that information is the basis for human learning in today's information-rich environments. It is associated with the common practice of information searching related to performing learning tasks in formal education contexts as well as work-life or everyday life. The majority of previous studies have focused on students' web searching behaviour and experience for their academic pursuits. It is widely known that web search engines are students' starting point for academic pursuits given such engines' speed, convenience and ease of use [27-29]. Students' mental model of search systems is a 'simple type-and-click operation' [30].

Several researchers have looked at when and how learning occurs in the search process for learning tasks. Vakkari et al. [16] examined relations between graduate students' problem stages in the course of writing their master's thesis proposals and the information sought, choice of search terms and relevance assessment. His study showed that students' level of knowledge about their topic predicts their ability to express search terms. According to his study, when students know less about the topic, they are likely to enter fewer, broader and more vague search terms as their queries. As Vakkari's study participants proceeded with their research process, the types of information sought and relevance criteria changed: the use of background and theoretical information declined and the importance of information types declined as predictors of relevance. More recently, Vakkari and Huuskonen [21] studied medical students' search behaviour and efforts with respect to a course assignment that required them to use the OVID-Medline biomedical search service. For this study, the authors distinguished search output (the products delivered by a system) and task outcome (the benefits the system produces). They found that effort in the search process degraded precision but improved task outcome. The findings of their study indicate that traditional effectiveness measures in information retrieval, such as recall and precision, need to be complemented with evaluation measures for search process and outcome.

A few studies have investigated learning as an outcome of searching. Hersh et al. [31] used a quiz approach to assess how well medical students were able to answer clinical questions after they used an information retrieval system. Their results showed that pre-searching scores on the test were low, but that searching enabled students to answer a higher proportion of questions. Based on the results, they proposed various methods as viable alternatives to measuring recall and precision. In Kammer et al.'s [32] study, participants were asked to formulate keywords and write a short summary to assess learning. The number of keywords submitted by participants was counted and their written summaries were assessed through topic coverage (the number of reasonable topics covered) and topic quality (each topic was rated on its quality). Jansen et al. [13] investigated Bloom's learning theory to characterize the information search process. Their study addressed two research questions: 'Are searching episodes learning events?' and 'Are searching characteristics with Anderson and Krathwohl's [9] taxonomy affected by the searcher's learning style?' (pp. 647-648). They analysed search tasks collected from participants and found that applying and analysing in the learning taxonomy take the most search effort in terms of queries per session, topics searched per session and total time searching. In conclusion, Jansen et al. claim that a learning theory can improve the information search process more than traditional paradigms of decisionmaking or problem-solving.

Journal of Information Science, 42(1) 2016, pp. 19-34 @ The Author(s), DOI: 10.1177/0165551515615841 
Over the past decade, exploratory search has emerged as an alternative perspective to characterize searching to foster learning and investigation [33]. A fundamental idea of exploratory search is that the primary function of the search system should not be to provide search results. Instead, it should be to help users explore, overcome uncertainty, and, eventually, learn [34]. Exploratory search would be a pertinent search strategy for searchers who are willing to learn through search because exploratory searchers utilize a combination of search and browsing behaviour to navigate, understand and better interpret the meaning of retrieved information [34]. Exploratory searches are characterized by multiple iterations and search results that require more cognitive processing than look-ups [8]. Exploratory searchers spend more time reading, comparing, and making qualitative judgments of information. In the exploratory model, learning is used in the general sense of developing new knowledge, including self-directed lifelong learning and professional learning as well as learning for academic pursuits [8].

Wilson and Wilson [14] were the first researchers to attempt to develop techniques to measure how much someone has learned as a result of exploratory search. Using Bloom's revised taxonomy [9], they developed three levels of measures for depth of learning: Quality of facts (D-Qual), Interpretation of data into statements (D-Intrp), and Use of critique (D-Crit). D-Qual focused on the quality of recalled facts by their usefulness, covering the 'remembering' and 'understanding' levels of Bloom's revised taxonomy. D-Intrp assessed how students synthesized facts and statements to draw conclusions and deductions (Bloom's analysing). D-Crit covered Bloom's concept of 'evaluating' by identifying statements that compared facts or used facts to raise more questions. The results of their study revealed that a 'one-size-fitsall' approach cannot be used to accurately measure the degree of learning because many variables may be confounded by other factors such as the length of the summaries provided by participants and their levels of prior knowledge.

\subsection{Learning to search}

This framework is closely connected to the concept of information literacy, implying learning how to critically seek, evaluate and use information for various search tasks. It takes the position that information literacy is essential for a student's academic success and provides a firm foundation for lifelong learning [35]. Information literacy is considered 'a survival skill in the information age' [36]. Researchers and practitioners in the field of information literacy believe that the ability to find, evaluate and use information effectively [37] is crucial for twenty-first-century learners. The American Association of School Librarians' Standards for the 21st-century learner [38] affirms the need for information literacy skill development in $\mathrm{K}-12$ education, asserting that 'the amount of information available to our learners necessitates that each individual acquire the skills to select, evaluate, and use information appropriately and effectively' [38: 3].

Research has found evidence of the ways and extent to which information literacy instruction can benefit people. Students who have demonstrated improved information literacy tend to have positive attitudes towards conducting research [39, 40] and greater confidence in their ability to perform such research in the future [41]. Top-tier students in college report experiencing formal information literacy instruction in grade school [17].

Unfortunately, there seems to be a mismatch between what traditional information literacy instruction programmes have emphasized and what students actually need to master competency for the purpose of gathering information in their learning environments. Most information literacy instructional programmes developed by librarians have focused on introducing library resources. Teachers value inquiry-based learning yet lack training in providing instructions on search strategies and evaluation of information in web searching. Students themselves seem to be ambivalent about information literacy instruction. Because they use search engines all the time, they think that finding information is something anyone can do [6]. They rely heavily on the open web for information and use web search engines as a starting place for class assignments $[42,43]$. They do not apply critical evaluation criteria such as authority, accuracy, objectivity, currency and coverage to the online sources they use for their coursework [44]. As their knowledge of search engines is limited, they assume that search engines 'understand' their queries and they are satisfied with the first few websites that search engines display [45]. Despite students' confidence in their search skills, tests of their knowledge of information literacy reveal that they overestimate their knowledge -they simply do not know what they do not know [6, 46-48].

\section{Searching as a learning process}

From the perspective of Searching as a Learning Process, searching is conceptualized as a process in which people engage in various search activities for learning, such as critically analysing information, bringing pieces of information together to create something new, evaluating and using information. What makes this perspective distinctive from Searching as a Learning Tool is that the former focuses on learning occurring during the search process rather than just the nature of search outputs (i.e. search results) and learning outcomes. One framework proposed in this section is 
comprehensive search, which suggests a paradigm shift from learning correct answers to learning based on a variety of information gathered and synthesized during the search process.

\section{I. Comprehensive search for critical and creative learning}

The perspective of Searching as a Learning Tool can be shifted towards a new perspective of Searching as a Learning Process through the concept of comprehensive search. Before describing the concept of comprehensive search, it is useful to review three modes of cognitive learning first. Receptive learning is defined as understanding, remembering and reproducing what is taught, such as facts, concepts, procedures and principles [49]. It corresponds to remembering and understanding in Bloom's taxonomy [50]. Critical learning is thinking differently with respect to an individual's own viewpoints by reviewing, criticizing and evaluating ideas gained from multiple sources. Critical learning includes applying, analysing and evaluating in Bloom's taxonomy. Creative learning is generating new ideas and designing new artefacts from what is learned. Although critical and creative learning involve higher cognitive activities in the learning taxonomy, this does not mean that they are more difficult than receptive learning. There might be easier critical learning assignments and more difficult receptive learning tasks. No matter how difficult the task is, it is within the receptive learning mode as long as its primary purpose is acquiring and receiving knowledge without any critical and creative evaluation. Each learning mode relates to the others, but they do not foster competencies in one another. For example, learning within only the receptive mode does not nurture critical thinking ability or creativity, no matter how intensively students study in receptive mode [49]. Cognitive processes and learning strategies differ across learning modes. As a result, each mode cultivates different types of competencies.

Comprehensive search depicts iterative, reflective and integrative search sessions that support critical learning and creative learning modes. In other words, comprehensive search refers to a variety of search activities that facilitate individuals' critical abilities and/or the development of new ideas directly. What is new about comprehensive search is that we are relating people's search behaviour to cognitive learning modes, while making distinctions among search activities supporting critical learning and creative learning. For instance, comprehensive search activities include critically evaluating the usefulness of information for tasks, assessing the credibility of information, differentiating the value of information across multiple sources of information and prioritizing information through comparison.

To some extent, comprehensive search shares similar characteristics with exploratory search. Marchionini [8] describes the following exploratory search activities that support learning: knowledge acquisition, comprehension, interpretation, comparison, aggregation, integration and socializing. According to Marchionini [8], investigative exploratory search involves multiple iterations over time to achieve analysis, synthesis, evaluation, discovery, planning/forecasting and transformation. Comprehensive search is distinct from exploratory search in two important ways. While exploratory search looks at learning and investigation as tasks that people are trying to achieve through a variety of search activities, comprehensive search captures the kinds of learning that occur during the search process. We claim that exploratory search is conceptualized from the perspective of searching to learn or investigate. In contrast, the concept of comprehensive search has been developed from the perspective of searching as a learning process emphasizing the interplay between cognitive learning and search activities. Another difference between exploratory search and comprehensive search is that the goal of comprehensive search is to support not only critical learning but also creative learning in terms of cognitive learning modes, whereas exploratory search is pertinent to supporting critical learning (e.g. comparison, integration, aggregation, analysis, synthesis, evaluation) and receptive learning (e.g. knowledge acquisition and comprehension).

Figure 1 illustrates how different kinds of search mode - fact finding, specified search and unfocused browsing - can be associated with the three cognitive learning modes explained above. Associations between search mode and cognitive learning mode produce different search paradigms. Look-up search refers to the fact-finding search that seeks accurate factual information. It mostly involves the receptive learning mode as the primary purpose is finding and acquiring existing information. In comparison, higher level cognitive activities such as analysing (differentiating, organizing, attributing), evaluating (checking, critiquing) and creating (generating, planning, producing) can be learned through comprehensive search, which serves as a learning process in itself. Students learn to differentiate similarities and differences among diverse perspectives, and to structure and hierarchize abundant materials. Students can also develop more flexible thinking skills as they consider diverse opinions and approaches on a topic rather than seeking correct answers. Furthermore, by comparing diverse logics, students may develop their own critical logic that they can then use to filter inaccurate information. This critical thinking ability is ultimately connected to creative learning, including generating, planning and producing new ideas and thoughts.

Table 1 shows how diverse learning and search behaviours relate to different cognitive learning modes. When people search in order to discover a new research theme, analyse and compare diverse perspectives about a topic, and/or

Journal of Information Science, 42(1) 2016, pp. 19-34 @ The Author(s), DOI: 10.1177/0165551515615841 


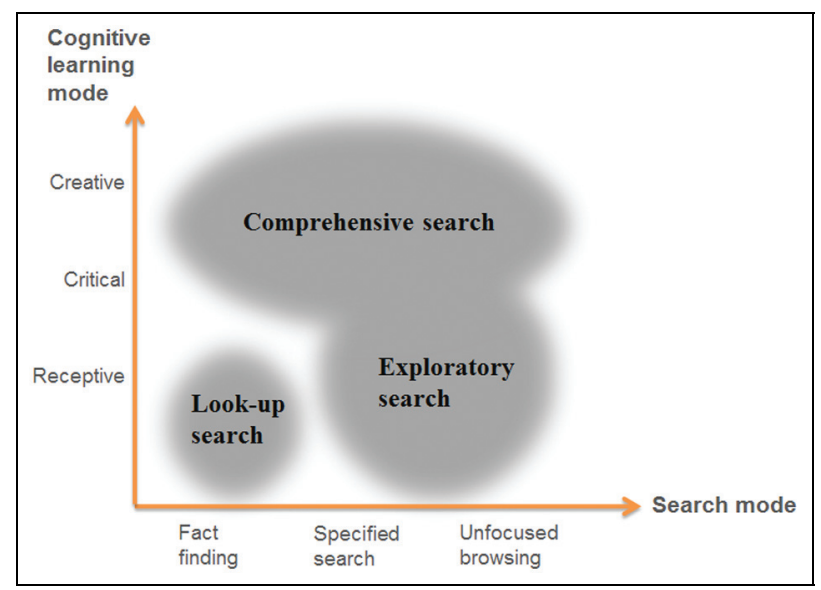

Figure I. A conceptual framework of association between search mode and cognitive learning.

Table I. Learning behaviour and search behaviour by cognitive learning taxonomy

\begin{tabular}{|c|c|c|c|}
\hline Cognitive learning mode & $\begin{array}{l}\text { Bloom's cognitive } \\
\text { learning taxonomy }\end{array}$ & Learning behaviour & Search behaviour \\
\hline Receptive & $\begin{array}{l}\text { remembering, } \\
\text { understanding }\end{array}$ & $\begin{array}{l}\text { recalling, presenting, identifying, } \\
\text { matching, labelling, comprehending, } \\
\text { demonstrating }\end{array}$ & $\begin{array}{l}\text { known-item searching, } \\
\text { specifying, modifying, obtaining, } \\
\text { selecting, acquiring, judging } \\
\text { relevance }\end{array}$ \\
\hline Critical & $\begin{array}{l}\text { applying, analysing, } \\
\text { evaluating }\end{array}$ & $\begin{array}{l}\text { separating, sorting, critiquing, } \\
\text { distinguishing, contrasting, defending, } \\
\text { attributing, probing, aggregating, } \\
\text { integrating, synthesizing }\end{array}$ & $\begin{array}{l}\text { evaluating usefulness, assessing } \\
\text { credibility, comparing, } \\
\text { extracting, differentiating }\end{array}$ \\
\hline Creative & creating & $\begin{array}{l}\text { hypothesizing, designing, discovering, } \\
\text { planning, producing, generating, } \\
\text { forecasting, inventing, composing, } \\
\text { revising, building }\end{array}$ & prioritizing, sense-making \\
\hline
\end{tabular}

Column I from Lee et al. [49] and column 2 from Bloom and Krathwohl [50].

brainstorm multiple solutions for a problem, they engage in distinct search and learning behaviours during the search process. Such behaviours may lead them to engage in critical rather than receptive learning.

\subsection{Implications of comprehensive search on epistemological beliefs and competency}

Epistemology is the branch of philosophy that investigates what knowledge is and how people know whether they know something [51]. Epistemic beliefs refer to a person's beliefs about the nature of human knowledge. The type of textbook learning featured in most primary and secondary education, for example, proceeds from the assumption that knowledge exists objectively, regardless of individuals' understanding. Thus, students are encouraged to identify correct answers rather than to develop their own ideas per se. From this epistemological standpoint, search activities are understood as seeking accurate information, and the purpose of a search system is to serve as an information retrieval tool.

In contrast, comprehensive search proceeds from a different set of epistemic beliefs that emphasize knowledge as constructed via diverse perspectives. Pedagogical techniques influenced by this epistemology encourage students to develop critical thinking skills, beyond simply finding a 'correct' answer. This helps students to develop logical thinking in order to compare and evaluate diverse perspectives. In this way, students' critical thinking capacity is enhanced. Search activities working from this epistemological standpoint will not necessarily or always present results linearly at the end of search process. Instead, knowledge expands as the student interacts with information in a variety of ways. In other words, knowledge is constructed by assimilation and accommodation of the learner's cognitive schema [52] as new information 
is incorporated into their existing knowledge structure. This is cognitive constructivism. From this perspective, information exploration during the search process is reconceptualized as an important part of the learning process itself.

A number of researchers have expressed concerns about the ways people search for information and interact with information on the Internet, pointing out that people tend to read online content too briefly and quickly [53]. Carr's book [54], The shallows, argues that online activities may be making our brains worse at reading long text and thinking deeply. Other researchers bemoan the behaviour of the 'Google Generation,' who are said to have 'little or no recollection of life before the web' [55] as they read or scan rapidly and make quick decisions without deliberation.

However, we need to question whether shallow learning can be easily written off as a negative and undesirable phenomenon. The competencies required in today's digital society are not necessarily identical to the ones required in the industrial age. Required competencies evolve over time. Previously, the ability to concentrate on one thing deeply for a long time without being distracted was, indeed, a required and valued competency. Further, it was a competency that would serve one well at schools designed according to the industrial age paradigm.

In today's digital era, with all the affordances of synchronous search technology, the human brain may well become familiarized with and adept at broad and relatively shallow searching. Furthermore, it might learn through this process to integrate more diverse information and knowledge, and create new ideas synthesizing that diversity. Instead of memorizing existing knowledge, knowing how to seek the best knowledge from the vastly expanded intellectual space of the Internet becomes a desirable competency in this century. Therefore, although some researchers are concerned that online activities hinder deep, reflective thinking and serve to deteriorate our memory [53, 54], we propose that the online search activities that we term 'comprehensive search' may nurture another capacity required in today's digital environment namely, the capacity for explorative and integrative thinking that compares diverse perspectives and generates new ideas. Put more simply, we argue that in the current learning environment breadth as well as depth of knowledge matters. Searching comprehensively is a pathway to integrating diverse information, which contributes, in turn, to a new paradigm of learning: critical and creative learning.

\section{Modelling and evaluating search-related learning}

Since web search engines are one of the primary access points people use to learn new information, analysis of web search engine logs provides one way to characterize, understand and assess the nature of search-related learning. The kind of interaction data present in search logs that captures events such as queries, clicks, mouse movement, dwell time and other traces of user behaviour provides an enriched representation of user goals and progress that is likely to be critical for implementing future comprehensive search engines. In this section, we discuss how search interaction data - even in its current limited form - can provide a rich source of implicit and explicit features that may be useful in supporting and assessing search-related learning, particularly in support of comprehensive search. We then summarize opportunities and challenges involved with inferring learning-related intent from search logs, and discuss some potential future directions.

First, we can begin by examining three key questions that are the subject of active research: (a) how do people use web search for learning tasks; (b) how can we estimate a search engine user's motivation to learn; and (c) how can we measure a user's change in expertise over time as they learn? Second, we discuss how progress on these questions informs how search technology might detect and support key learning activities in comprehensive search.

\section{I. Inferring the presence and nature of learning-related tasks from search logs}

How do people use web search for learning tasks? As described earlier, learning-related search tasks can be complex, requiring multiple queries and significant time spent searching and browsing. There have been few published studies of how users engage in such tasks based on commercial search engine data. However, one study by Bailey et al. [56] described a task taxonomy of web searches - based on analysis by human judges of 4 months of search log data from Google, Yahoo and Bing - that included some learning-related tasks, including topic exploration, fact-finding and procedural learning. A second study by Eickhoff et al. [57] included an automated log analysis that focused specifically on the fraction of sessions that potentially involved a procedural or declarative knowledge intent. The relevant results from both studies are summarized in Table 2. Both studies found that learning-related tasks accounted for a non-trivial proportion of all search sessions, as shown in the 'percentage of sessions' column in Table 2 . In line with the task study by Bailey et al. [56], Eickhoff et al. [57] found that about 3\% of sessions had an explicit knowledge-acquisition intent that was either procedural or declarative. Moreover, reflecting the complex, multi-step nature of many learning-related tasks, while the absolute percentage of total sessions for the learning tasks in Table 2 is around 20\%, these tasks accounted for a disproportionally larger fraction of time spent searching.

Journal of Information Science, 42(1) 2016, pp. 19-34 @ The Author(s), DOI: 10.1177/0165551515615841 
Table 2. Time spent on learning-related search tasks

\begin{tabular}{|c|c|c|c|c|}
\hline Task & $\begin{array}{l}\text { Percentage } \\
\text { of sessions }\end{array}$ & $\begin{array}{l}\text { Average queries } \\
\text { per task }\end{array}$ & $\begin{array}{l}\text { Average events per task } \\
\text { (browse and search) }\end{array}$ & $\begin{array}{l}\text { Average task length } \\
\text { (minutes) }\end{array}$ \\
\hline $\begin{array}{l}\text { Discover more information } \\
\text { about a specific topic }\end{array}$ & $8.0 \%$ & 6.8 & 24.8 & 13.5 \\
\hline Find a specific fact & $7.0 \%$ & 3.2 & 40.0 & 7.9 \\
\hline Find facts about a person & $1.8 \%$ & 6.9 & 18.9 & 4.8 \\
\hline Learn how to perform a task & $2.0 \%$ & 13.0 & 11.9 & 8.5 \\
\hline Do education-related tasks online & $1.5 \%$ & - & - & - \\
\hline Procedural knowledge intent & $1.7 \%$ & 4.1 & - & 10.0 \\
\hline Declarative knowledge intent & $1.4 \%$ & 6.3 & - & 16.7 \\
\hline
\end{tabular}

Rows I-5 from Bailey et al. [56] and rows 6-7 from Eickhoff et al. [57]. A blank ( - ) cell indicates specific numbers were not reported for that intent/task.

Raman et al. [58] had a similar finding for intrinsically diverse (ID) search tasks, which are exploratory web searches whose intent is to learn about multiple aspects of a specific topic. Given that ID sessions require multiple queries, Raman et al. hypothesized that ID sessions accounted for a disproportionately larger fraction of time spent searching by all users. To test this, they estimated the time a user spent in a session by the elapsed time from the first query to the last action (i.e. query or click). Sessions with a single query and no clicks were assigned a constant duration of $5 \mathrm{~s}$. (The time in session includes the whole session once an ID task was identified in that session.) While ID sessions with at least two distinct exploratory aspects represented only $1.0 \%$ of all sessions, they accounted for more than $4.3 \%$ of total time spent searching, showing the significant role that ID sessions play in overall search activity. The study by Raman et al. did not focus on more complex ID information seeking, such as tasks that span multiple sessions, and so the true percentage associated with ID tasks is likely to be larger.

These studies reflect the two basic approaches - manual vs automated - that are used to infer user intent from search logs. Manual approaches rely on either self-reported intent indicators generated by the users themselves (e.g. via an affordance in the search user interface), or intent descriptions generated by human judges who try to deduce intent from a post-hoc analysis of search $\log$ behaviour data. The latter was the approach used by Bailey et al. [56], following an earlier method developed by Rose and Levinson [59], to determine intent categories (e.g. 'Discover more information about a specific topic'). The log behaviour data that is used as the basis for these judgments includes (a) the queries issued by a user in a session, (b) the results returned by the search engine, (c) the results clicked by the user, and (d) further queries or other actions taken by the user. This data also potentially includes additional resources such as the actual content of the pages returned in the search results, along with meta-data computed from this content, for example, the page's reading level or topic category. On the other hand, automated approaches [57, 58] typically rely on a representative sample of user behaviour data for which some information about intent has been identified by human judges, but then apply this initial set of labelled data to train a statistical model that attempts to automatically classify intent for future instances of user behaviour.

In the case of learning-related search, one approach that automated methods have used to operationalize indicators of learning intent is to use the type of knowledge offered by a website's educational content as a proxy for a learning intent label. For example, in Eickhoff et al. [57] a user's visitation of a site specializing in procedural 'how-to' descriptions (e.g. ehow.com) was assumed to indicate a procedural learning intent, while Wikipedia visits were assumed to be indicative of intent to acquire declarative 'fact-based' knowledge. These implicit intent labels were then used as the basis for characterizing features of queries and patterns of search behaviour that were associated with each type of learning intent. Similarly, in Raman et al. the nature and breadth of topic categories for the content covered during a search is used as a proxy indicator of the user's desire to explore different aspects of a topic. Other examples include using reading-level features associated with motivation (Section 4.2) and features of site topic focus and difficulty to measure change in expertise over time (Section 4.3).

While these operational choices for learning indicators lead to richer representations of user behaviour while learning, they are not without their limitations. First, the use of proxy indicators of learning, such as representing a user's content intent by the content type they read, assumes that what the user is finding is what they intended to find, and that what they intend to find represents what they intend to learn. These assumptions often do not hold in practice: users may have a variety of goals when looking at all or part of a particular page. Users may also be restricted by the results of an ineffective search engine or choice of search strategies for a given learning goal, leading to less relevant content being 
perused, creating an inaccurate set of proxy learning indicators. We discuss future directions researchers are pursuing for overcoming some of these issues in Section 5.4. Second, search log data may provide a limited window on what the user is seeing, paying attention to and expressing about their need. In many cases, for example, the search log behaviour of a user who is struggling can appear to be very similar to that of a user who is successful in their efforts [60]. In such scenarios, researchers have successfully developed and applied classifiers that detect characteristic query reformulation patterns of struggling users in order to distinguish success from frustration [60]. Finally, an additional set of limitations stems from the nature of search log data itself. Even assuming a perfect ability to infer intent from data, obtaining complete and correct user behaviour data for individuals can be problematic. Attribution of search activity to a specific user is usually performed using an auto-generated unique identifier based on Web cookies or a browser instance. However, multiple people searching on the same machine is common: a recent study by White et al. estimated that $56 \%$ of unique identifiers in a search log actually comprised activity from multiple searchers [61]. Conversely, a single user's log activity may be split across machines or devices, making a complete picture of that individual's behaviour more difficult to obtain. This problem is being addressed in part by the development of automatic methods to attribute activity to the correct user [61].

In short, there are many sources of potential noise and uncertainty in attempting to infer the latent intent or mental state of the user from a limited set of online observations. While this is indeed a difficult challenge, the examples cited above, along with future work discussed below, show that researchers are making progress in using machine learning and deeper natural language processing approaches to at least identify high-precision subsets of cases where algorithms can achieve an effective level of confidence in their predictions of learning intent and progress.

\subsection{Estimating motivation to learn while searching}

Given a particular web search-based learning task, other recent work has attempted to assess the motivation that users exhibit when attempting to complete information-seeking tasks. In a study by Kim et al. [62], the authors attempted to capture a user's motivation to find important information by estimating the effort they undertook to get it, via their reading stretch behaviour, whereby users engage in short-term search activity that involves searching and browsing documents far above their 'typical' reading level. To detect reading stretch behaviour, Kim et al. first computed a text readability score for every web page accessed in the search log, using the robust statistical approach given by CollinsThompson and Callan [63]. With these readability scores, they were able to estimate a user's typical reading level profile over a 2 month period by computing the distribution of readability scores over past clicked documents. Next, they identified search sessions in which the average reading level of clicked documents was at least four US grade levels higher than the average reading level of that same user's reading profile. By comparing normalized word histograms from titles of clicked pages in 'stretch' sessions with titles in all search sessions, they elicited the topics and tasks that were highly associated with stretch reading behaviour. These tasks corresponded to high-motivation scenarios in which users were trying to learn about areas such as medical tests and procedures, college entrance tests and financial aid options, filling out government forms, finding technical solutions to computer crashes and virus infections, and looking for a new job.

\subsection{Measuring change in expertise over time}

Learning and expertise are closely connected: expertise is an evolving characteristic of users that reflects learning over time, rather than being a static property. Wildemuth [64] examined how a user's domain expertise, that is, their knowledge and experience in a particular profession or discipline, was reflected in their choice of search strategies. She found that domain novices tended to exhibit increasingly similar search strategies to those of more expert users as the novices learned more about the topic. Other previous work on domain knowledge and expertise [65] has characterized domain expertise and search behaviour in terms of metrics that can be derived from search logs, such as:

\footnotetext{
Website visitation: Experts tend to visit different and more diverse websites in a subject area than novices. One measure of this is the average number of unique top-level websites on a search result page (SERP) or across clicked documents.

Focus: Experts tend to explore more narrow topical spaces than non-experts. The degree to which a SERP is covered by a single topic is one measure of focus. Topics may be derived based on existing class hierarchies such as the Open Directory Project.

Entropy: While focus measures degree of coverage by a single topic, entropy captures diversity across multiple topics on search results pages. Experts typically have higher focus, less diversity, and thus, lower entropy across topics.

Branchiness: White et al. [65] found that domain experts tend to follow more 'branchy' paths than novices, returning to a previously visited point and then following a new unexplored direction. Specific path features include the search depth and quality of results along typical paths.
}

Journal of Information Science, 42(1) 2016, pp. 19-34 @ The Author(s), DOI: 10.1177/0165551515615841 
Dwell time: This is the time a user spends reading a search result document. White et al. [65] found that experts tend to spend less time per page during a search, perhaps because they are more adept at making assessments of technical or specialized content. A more general category of features measures the pace of interaction for search and browsing (e.g. time until the first question is asked, or time until the first document is saved).

Query and session complexity: The use of domain-specific, technical, or specialized terms in queries can be associated with expert vs non-expert searchers. More generally, characteristics such as the number of queries submitted and query reformulation patterns may also be used.

White et al. [65] identified expert users in medicine, finance, law and computer science by their visitation patterns to a pre-determined set of specialist websites, which were identified in turn from discussion with domain experts in each subject area. The non-expert users selected for comparison were those who also showed interest in the same topics according to their search behaviour. In related work, Zhang et al. [66] characterized domain expertise in terms of similar classes of features derived from search behaviour.

Previous work on modelling domain expertise in searching typically focused on longer-term behaviour patterns across sessions. However, in one of the first large-scale search log-based studies to examine session-level features of tasks where people are explicitly searching for new knowledge, Eickhoff et al. [57] looked at within-session changes for these expertise metrics. They focused on two specific types of knowledge acquisition: procedural knowledge (how to do something) vs declarative knowledge (knowing facts about something). The authors found evidence both for learning progress within single sessions and for persistence of learning across sessions. Significant proportions of new query terms came from result page snippets and recently visited pages, showing that the search process itself contributed to augmenting the user's domain knowledge.

\subsection{Log-based analysis and metrics for comprehensive search}

In general, current web search engines provide a rather limited interface, and thus, a correspondingly limited set of interaction events and content for logging. Although users interact with web search interfaces for the purpose of comprehensive search - evaluating usefulness, assessing credibility, comparing multiple sources and differentiating value of information - such interactions are rarely captured with current interfaces for web search engines. Thus, we envision that progress in machine learning and natural language processing algorithms for tackling the challenges of current search $\log$ analysis, as described above, will provide a good foundation for further advances in understanding human searching as a learning process. This progress will be especially effective when applied to the collection of rich search trails and analysis of interactions that will be enabled by the affordances present in the next generation of user interfaces that support comprehensive search for critical and creative learning.

The log-based analysis and metrics described above are well-suited to being extended to support comprehensive search. In one example, users' search trails to and from web documents, which were derived in a limited form from traditional search logs to enable expertise modelling as described above, could be extended in two ways. First, additional events based on comprehensive search activity could capture new sources of evidence from users, for example, user judgements about evaluation, credibility or comparison of multiple sources. Second, new types of relationships between documents that constitute a search trail, such as the set of documents that give credible alternative views for a given document, could be added to the search trail representation. This combination of new user events and new document relationship types could enable comprehensive search engines to use the resulting log data for learning the association between properties of comprehensive search trails and the estimated usefulness of documents for different learning outcomes.

\section{Agendas for future research}

Based on the literature review carried out in this paper, we suggest the following future research directions and agendas.

\section{I. Developing a search system that supports sense-making and enhances learning}

Search systems need to be reconfigured to supporting more diverse types of learning beyond knowledge acquisition. As we discussed in the Introduction section, current search systems are optimized for look-up tasks and not well-suited for learning and investigative tasks [8]. Marchionini [8] and other researchers [33, 34] have proposed exploratory search as a framework that could support search activities for the purpose of learning and investigation. We would go even further, arguing that search systems could be transformed as a learning technology that could support critical and creative learning through various kinds of search behaviour, such as evaluating the usefulness of information critically, differentiating, 
monitoring, tracking, prioritizing and sense-making. While engaging in the diverse behaviour that comprises comprehensive search, people accomplish those learning activities described in Bloom's taxonomy as higher-level learning, including organizing ideas, identifying and analysing patterns, comparing different ideas, integrating ideas, and eventually, creating new ideas. This research direction moves away from conceptualizing search systems as a 'tool' for learning. Instead, this direction emphasizes the learning processes, experiences and outcomes involved during and after the search process to support critical and creative learning.

Another concept that could usefully inform the design of search systems is that of sense-making. The sense-making process is about creating knowledge structures and making sense of the data/information that has been acquired through the information seeking task [67]. Generally, such representations use textual representations and maps, diagrams and tables or other modalities to organize and structure information in order to make sense of it [68]. The sense-making process is about encoding information into external representations in order to answer complex, relational, interlinked, contextual and task-specific questions. A new search system could support learning by offering more interactive functions such as tagging for annotation or tracking individuals' search history so that a learner could return to a particular learning point. In addition, a system could provide new features that allow learners to reflect upon their own learning process and search outcomes, thus facilitating the development of critical thinking skills.

\subsection{Supporting effective user interaction for searching as learning}

One approach to take in future research is to design search interfaces to better support learning with respect to the search process [69]. It is well known that information objects encountered during the search process could trigger the searcher to shift their search tasks or intentions [22]. O'Day and Jeffries [70] found that search results for a goal tend to trigger new goals; they concluded that one of the main values of searching was, thus, the very process of acquiring information and accumulating learning. Therefore, new tools might be designed to facilitate learning by prompting with additional query terms or asking questions for critical thinking.

Marchionini et al. [71] present a framework that describes a set of six different user interface views they call agileviews. Agileviews consist of the following views in which a variety of interaction with information can take place: primary views, overviews, previews, reviews, peripheral views and shared views. The primary view shows the documents that have been accessed, and the list of the search results. Here the user may not only see a ranked list of hits. According to Table 1, the user can at this stage be involved in learning behaviour like recognizing or recalling, matching and comprehending. The overviews may facilitate different kinds of starting points for interacting and utilizing the information for the user. The overviews will also orient the user to the choices that are available and thus, start learning activities such as labelling, separating and sorting (Table 1). Marchionini's [71] previews interface shows the user what will happen if a certain choice is made. The learning behaviours that could be supported here are probing, planning, aggregating and integrating (Table 1). This could then be used for more informed decisions of what to do next [68]. Reviews allow for revisiting past choices, essentially providing search history, and this may support learning activities such as recalling, identifying, sorting, planning and revising (Table 1). Next, peripheral views show 'background' information that eventually may become of interest. This type of information is not the current focus of attention for the user. Learning behaviour that could be supported by this activity includes contrasting and monitoring. Finally, shared views can inform and show past and current state of search process that other people are involved in [72]. This is a very important view that may be related to learning behaviours such as comprehending, critiquing, contrasting and discovering (Table 1).

As mentioned above, browsing can be considered as part of the search process. Browsing strategies involve the process of following a chain of links, but also jumping between different views [73]. In this way browsing could be considered as a casual and undirected exploration of navigation structures (mental models and cognitive representations of information). In most cases, these navigation (browsing) structures are automatically or manually defined. Documents are then assigned to these labels/categories. Hearst [73] describes a set of interfaces for grouping search results and collections by flat categories, hierarchical categories and also faceted categories, which can have implication for supporting learning behaviours

In agreement with Hearst [73], the agileviews framework emphasizes the need to support dynamic interaction among the different types of views to support search strategies. We argue that, when designing these navigational structures as well as designing the search functionalities, support for learning behaviours should be acknowledged.

\subsection{Providing an inquiry-based information literacy tool within a search system}

The third research direction is to develop a new kind of information literacy programme that integrates information literacy instructions into the design of a search system. To this end, we could consider developing a search system that

Journal of Information Science, 42(1) 2016, pp. 19-34 @ The Author(s), DOI: 10.1177/0165551515615841 
employs a scaffolding-guided inquiry framework. Scaffolding refers to the practice of supporting learners by providing targeted assistance, either technical or social (through adults or capable peers), in order to help learners engage in tasks that are beyond their current level of proficiency [74]. This new information literacy tool could also use 'guided inquiry' [75], which emphasizes open-ended questions that motivate students to take more active ownership of their own learning through exploratory research processes. The guided inquiry process has eight phases: open the inquiry; immerse in the experience; explore interesting ideas; identify the inquiry question; gather important information; create to communicate; share learning; and evaluate achievement of learning goals [76]. Through the inquiry process, students are actively engaged in the subject content, pursuing a range of specific questions and attaining a deeper and broader understanding of the content. Research questions to be asked towards this research direction include: (a) what features and functionality of the scaffolding-guided inquiry tool are effective at helping students to engage in active searching and sense-making; (b) to what extent do students learn more broadly by being actively engaged in the search process; and (c) to what extent do students develop critical learning skills through scaffolding-guided inquiry? This tool could also involve cognitive and metacognitive strategies to support students with various aspects of searching, such as helping students track their search progress with respect to their goals and helping them reflect on important aspects of their searching process by using tagging features. By incorporating the design approach developed in scaffolding-guided inquiry [77], the purpose of developing an information literacy tool is not to improve students' searching skills but to improve their critical thinking skills.

\subsection{Future directions for assessing learning from online search behaviour}

Focusing on the goal of comprehensive search will require research advances in most, if not all, of the key areas discussed here. In particular, modelling and assessing comprehensive search progress will require richer representations of learning and expertise that capture not only the nature and progress of domain expertise over time, but also potentially how this additional expertise is acquired, for example, if diverse perspectives are encountered along the learning path, or if multiple sources of evidence are gathered in support of a conclusion. Recent research that has mined the rich user interaction evidence in search trails [78] from log data may be one approach to characterizing important features of the learning process during search.

In order to provide search results that are most appropriate for a specific user's desired level of understanding, researchers have also begun investigating search engine ranking algorithms that account for the complexity of content compared with the domain expertise of learners. These are based on user profiles and ranking algorithms that have been trained on features derived partly from extensive log data. A recent example of this is the study by Collins-Thompson et al. [79], which found that text readability was a valuable relevance signal for web search, leading to significantly improved rankings. In related work, Tan et al. [80] defined a comprehensibility measure of content posted on the Yahoo! Answers question-answering site in order to provide personalized ranking that ranked simple vs complex technical answers depending on the user's domain expertise. In another promising direction, researchers have begun exploring additional sources of behavioural data, for example, eye-tracking devices [81] that can augment log files with gaze position and movement data to provide a more complete view of where and how the user is focusing their attention and learning effort.

In sum, researchers are making promising advances towards models of learning intent, motivation, domain expertise and content complexity that can enable richer representations of queries, users and content for assessing learning opportunities and outcomes. Furthermore, these findings provide early evidence that implicit and explicit signals from search interaction can be used to identify learning tasks, assess motivation and predict the degree and growth of a user's domain expertise. In future work, what we learn from log-based assessment measures - while not without its limitations - could be used as an additional rich source of evidence for improving future result quality and overall search experience for personalized, learning-related search tasks.

\section{Conclusion}

The analysis of previous studies on searching and learning reveals that the association between searching and learning was often unclear and the association between these two concepts has not been the focus. In this paper, we did not attempt to provide a definitive statement characterizing the relationship between searching and learning. Rather, we presented multiple perspectives and empirical studies that demonstrate the complexity and 'messiness' of the association of searching and learning. We then proposed a new perspective on searching as a learning process focusing on comprehensive search. We also discussed the potential of search interaction data to support and assess search-related learning. Finally, we presented three research directions that could be pursued in future research. 
Based on the critical reviews we have conducted, we offer the following summaries about searching as learning:

(1) Perspectives - it is important to distinguish different kinds of constructs of learning in searching. When information is defined as a thing [82] that humans seek, evaluate and use, searching is conceptualized as a learning tool and assessment of learning is often made based on search outputs. When information is considered as knowledge or belief from a constructivist epistemology, searching is reconceptualized as a learning process best measured in terms of conceptual changes in existing knowledge structure and beliefs, as well as the outcomes for search tasks.

(2) Evaluation - previous research provides early evidence that learning-related tasks account for a significant proportion of all web search sessions, and that implicit and explicit signals from search interaction can be used to identify learning tasks, assess motivation and predict the degree and growth of a user's domain expertise. We envision that this growing body of research that seeks to understand and support search users by leveraging their interaction patterns and history will also have fruitful applications to tasks and assessment involved in comprehensive search.

(3) System - recent work has demonstrated that models of domain expertise and content complexity can enable the assessment of learning opportunities and outcomes. Such modelling could serve as the basis for developing an innovative search system that supports people's learning through comprehensive searching.

(4) Interface - we discussed how earlier research in the areas of interaction design and information seeking and retrieval, such as user interfaces that provide rich sets of search views and browsing support - and ways for search and browsing to interact - are well-aligned with different comprehensive search tasks. We envision comprehensive search systems being built by bringing together these elements in novel ways to provide information spaces that facilitate explicit and implicit learning behaviours. Moreover, we foresee that the search log data gathered from users interacting with these new interface elements and affordances will provide novel data mining opportunities on which current log analysis techniques for understanding and supporting learning can build.

At least three research communities - information behaviour, information literacy and information retrieval - within the field of information science have long investigated the intersection of searching and learning. In order to advance research on searching as learning, these research communities need to engage in more communication and collaboration. In addition, theoretical and empirical research conducted in other disciplines such as learning sciences, machine learning, instructional technology and educational psychology will also play an important role in helping us develop innovative search technologies of the future. We hope that this paper has successfully demonstrated that the pursuit of searching as learning as a distinct research agenda is a challenging, but worthwhile problem facing information science researchers.

\section{Funding}

This research received no specific grant from any funding agency in the public, commercial, or not-for-profit sectors.

\section{References}

[1] Beheshti J, Bilal D, Druin A et al. Testing children's information retrieval systems: Challenges in a new era. Proceedings of American Society for Information Science and Technology 2010; 47(1): 1-4.

[2] Dhillon MK. Online information seeking and higher education students. In: Chelton M and Cool C (eds), Youth informationseeking behavior II: Context, theories, models, and issues. Lanham, MD: Scarecrow Press, 2007, pp. 165-205.

[3] Druin A, Foss E, Hatley L et al. How children search the internet with keyword interfaces. In: Proceedings of the 8th international conference on interaction design and children. New York: ACM, 2009, pp. 89-96.

[4] Large A, Nesset V and Beheshti J. Children as information seekers: what researchers tell us. New Review of Children's Literature and Librarianship 2008; 14(2): 121-140.

[5] Pan B, Hembrooke H, Joachims T et al. In Google we trust: Users' decisions on rank, position, and relevance. Journal of Computer-Mediated Communication 2007; 12(3): 801-823.

[6] Gross M and Latham D. What's skill got to do with it?: Information literacy skills and self-views of ability among first-year college students. Journal of the American Society for Information Science and Technology 2012; 63(3): 574-583.

[7] Rieh S, Kim Y and Markey K. Amount of invested mental effort (AIME) in online searching. Information Processing and Management 2012; 48(6): 1136-1150.

[8] Marchionini G. Exploratory search: From finding to understanding. Communications of the ACM 2006; 49(4): 41-46.

[9] Anderson L and Krathwohl D. A taxonomy for learning, teaching, and assessing. New York: Longman; 2001.

[10] Allan J, Croft B, Moffat A. et al. Frontiers, challenges, and opportunities for information retrieval: Report from SWRIL 2012 The Second Strategic Workshop on Information Retrieval in Lorne. ACM SIGIR Forum 2012; 46(1): 2-32. 
[11] Agosti M, Fuhr N, Toms E and Vakkari P (eds). Evaluation of methodologies in information retrieval. Dagstuhl Reports 2013; $3(10)$.

[12] Limberg L and Alexandersson M. Learning and information seeking. Encyclopedia of Library and Information Sciences 2010: 3252-3262.

[13] Jansen BJ, Booth D and Smith B. Using the taxonomy of cognitive learning to model online searching. Information Processing and Management 2009; 45: 643-663.

[14] Wilson MJ and Wilson ML. A comparison of techniques for measuring sensemaking and learning within participant-generated summaries. Journal of the American Society for Information Science and Technology 2013; 64 (2): 291-306.

[15] Kuhlthau C. Seeking meaning. Westport, CT: Libraries Unlimited, 2004.

[16] Vakkari P, Pennanen M and Serola S. Changes of search terms and tactics while writing a research proposal. Information Processing and Management 2003; 39(3): 445-463.

[17] Gross M and Latham D. Attaining information literacy: An investigation of the relationship between skill level, self-estimates of skill, and library anxiety. Library and Information Science Research 2007; 29(3): 332-353.

[18] Neuman D. Learning in information-rich environments. New York: Springer, 2011.

[19] American Association of School Librarians and Association for Educational Communications and Technology. Information power: Building partnerships for learning. Chicago, IL: American Library Association, 1998.

[20] Vakkari P. Changes in search tactics and relevance judgements when preparing a research proposal: A summary of the findings of a longitudinal study. Information Retrieval 2001; 4(3-4): 259-310.

[21] Vakkari P and Huuskonen S. Search effort degrades search output but improves task outcome. Journal of the American Society for Information Science and Technology 2012; 63(4): 657-670.

[22] Xie I. Interactive information retrieval in digital environments. Hershey, PA: IGI, 2008.

[23] Bloom B. Taxonomy of educational objectives. New York: McKay, 1956.

[24] Arguello J, Wu W-C, Kelly D and Edwards A. Task complexity, vertical display and user interaction in aggregated search. In: Proceedings of the ACM SIGIR conference on research and development in Information Retrieval, 2012, pp. 435-444.

[25] Wu W-C, Kelly D, Edwards A and Arguello J. Grannies, tanning beds, tattoos and NASCAR: Evaluation of search tasks with varying levels of cognitive complexity. In Proceedings of the 4th information interactions in context (IIiX), 2012, pp. $254-257$.

[26] Kelly D, Arguello J, Edwards A and Wu W-C. Development and evaluation of search tasks for IIR experiments using a cognitive complexity framework. In: Proceedings of the international conference on the theory of information retrieval (ICTIR), 2015, pp. 101-110.

[27] Head A. Beyond Google: How do students conduct academic research? First Monday 2007; 12(8).

[28] Head A and Eisenberg M. Truth be told: How college students evaluate and use information in the digital age. SSRN 2281485 2010 .

[29] Fast K and Campbell D. 'I still like Google': University student perceptions of searching OPACs and the web. In: Proceedings of American Society for Information Science and Technology, 2004, pp. 138-146.

[30] Chung J and Neuman D. High school students' information seeking and use for class projects. Journal of the American Society for Information Science and Technology 2007; 58(10): 1503-1517.

[31] Hersh WR, Elliot DL, Hickam DH et al. Towards new measures of information retrieval evaluation. In: Proceedings of the ACM SIGIR conference on research and development in information retrieval, 1995, pp. 164-170.

[32] Kammerer Y, Nairn R, Pirolli P and Chi EH. Signpost from the masses: Learning effects in an exploratory social tag search browser. In: Proceedings of the SIGCHI conference on human factors in computing systems, 2009, pp. 625-634.

[33] White R and Roth R. Exploratory search: Beyond the query-response paradigm. Synthesis Lectures on Information Concepts, Retrieval, and Services 2009; 1(1): 1-98.

[34] White R and Drucker S. Supporting exploratory search. Communications of the ACM 2006; 49(4): 36-39.

[35] Brown C and Krumholz L. Integrating information literacy into the science curriculum. College and Research Libraries 2002; 63(2): 111-123.

[36] Oblinger D and Hawkins B. The myth about student competency. Educause Review 2006; 24(1): $12-13$.

[37] Association of College and Research Libraries. Information literacy competency standards for higher education. Chicago, IL: Association of College and Research Libraries, 2000.

[38] American Association of School Librarians. Standards for the 21st-century learner. Chicago, IL: American Association of School Librarians, 2007.

[39] Daugherty $\mathrm{T}$ and Carter E. Assessment of outcome-focused library instruction in psychology. Journal of Instructional Psychology 1997; 24(1): 29-33.

[40] Wang R. The lasting impact of a library credit course. Portal: Libraries and the Academy 2006; 6(1): 79-92.

[41] Markey K, Leeder C and Rieh S. Designing online information literacy games students want to play. Lanham, MD: Rowman \& Littlefield, 2014.

[42] Madden M and Jones S. The internet goes to college. Pew Internet and American Life Project, 2002.

[43] Head A and Eisenberg M. How college students use the Web to conduct everyday life research. First Monday 2011; 16(4).

[44] Julien H and Barker S. How high-school students find and evaluate scientific information: A basis for information literacy skills development. Library and Information Science Research 2009; 31(1): 12-17. 
[45] Rowlands I, Nicholas D, Williams P et al. The Google generation: The information behaviour of the researcher of the future. Aslib Proceedings 2008; 60(4): 290-310.

[46] Brown C, Murphy T and Nanny M. Turning techno-savvy into info-savvy: authentically integrating information literacy into the college curriculum. The Journal of Academic Librarianship 2003; 29(6): 386-398.

[47] Jenson J. It's the information age, so where's the information? Why our students can't find it and what we can do to help. College Teaching 2004; 53(3): 107-112.

[48] Latham D and Gross M. Broken links: Undergraduates look back on their experiences with information literacy in k-12 education. School Library Media Research, http:/www.eric.ed.gov/ERICWebPortal/search/detailmini.jsp?_nfpb=true\&_\&ERICExt Search_SearchValue_0=EJ823031\&ERICExtSearch_SearchType_0=no\&accno=EJ823031 (2008, accessed 14 April 2015).

[49] Lee H, Lee J, Makara K et al. Does higher education foster critical and creative learners? An exploration of two universities in South Korea and the USA. Higher Education Research and Development 2014; 34(1): 131-146.

[50] Bloom B and Krathwohl D. Taxonomy of educational objectives book 1: Cognitive domain. Reading, MA: Addison Wesley, 1984.

[51] BonJour L. Epistemology: Classic problems and contemporary solutions. Lanham, MD: Rowman \& Littlefield, 2002.

[52] Powell KC and Kalina CJ. Cognitive and social constructivism: Developing tools for an effective classroom. Education 2009; 130(2): 241.

[53] Rosenwald M. Serious reading takes a hit from online scanning and skimming, researchers say. The Washington Post, http:// www.washingtonpost.com/local/serious-reading-takes-a-hit-from-online-scanning-and-skimming-researchers-say/2014/04/06/ 088028d2-b5d2-11e3-b899-20667de76985_story.html (2014, accessed 14 April 2015).

[54] Carr N. The shallows. New York: W.W. Norton, 2010.

[55] Rowlands I and Nicholas D. Information behaviour of the researcher of the future. Centre for Information Behaviour and the Evaluation of Research, http://www.webarchive.org.uk/wayback/archive/20140614113419/http://www.jisc.ac.uk/media/documents/programmes/reppres/gg_final_keynote_11012008.pdf (2008, accessed 14 April 2015).

[56] Bailey P, Chen L, Grosenick S et al. User task understanding: a web search engine perspective. In: NII Shonan workshop, 8-11 October 2012, Shonan, Japan, http://research.nii.ac.jp/ntcir/shonan/Bailey-NIIShonan-UserTaskUnderstanding.pdf

[57] Eickhoff C, Teevan J, White R et al. Lessons from the journey: A query log analysis of within-session learning. In: Proceedings of the seventh ACM international conference on web search and data mining (WSDM), 2014, pp. 223-232.

[58] Raman K, Bennett PN and Collins-Thompson K. Understanding intrinsic diversity in web search: Improving whole-session relevance. ACM Transactions on Information Systems 2014; 32(4): 1-45.

[59] Rose DE and Levinson D. Understanding user goals in web search. In: Proceedings of the 13th international conference on the World Wide Web (WWW'04), 2004, pp. 13-19.

[60] Odijk D, White RW, Awadallah AH and Dumais S. Struggling and success in web search. In: Proceedings of the 24th ACM international conference on information and knowledge management (CIKM 2015), 2015.

[61] White RW, Hassan A, Singla A and Horvitz E. From devices to people: attribution of search activity in multi-user settings. In: Proceedings of the 23rd international conference on World Wide Web (WWW'14), 2014, pp. 431-442.

[62] Kim J, Collins-Thompson K, Bennett PN et al. Characterizing web content, user interests, and search behavior by reading level and topic. In: Proceedings of the fifth ACM international conference on web search and data mining (WSDM), 2012, pp. 213-222.

[63] Collins-Thompson K and Callan J. Predicting reading difficulty with statistical language models. Journal of the American Society for Information Science and Technology 2005; 56(13): 1448-1462.

[64] Wildemuth BM. The effects of domain knowledge on search tactic formulation. Journal of the American Society for Information Science and Technology 2004; 55(3): 246-258.

[65] White RW, Dumais ST and Teevan J. Characterizing the influence of domain expertise on web search behavior. In: Proceedings of the second ACM international conference on web search and data mining (WSDM), 2009, pp. $132-141$.

[66] Zhang X, Cole M and Belkin N. Predicting users' domain knowledge from search behaviors. In: Proceedings of the 34th international ACM SIGIR conference on research and development in information retrieval, 2011, pp. 1225-1226.

[67] Russell DM, Stefik MJ, Pirolli P and Card SK. The cost structure of sensemaking. In: Proceedings of the INTERACT '93 and CHI'93 conference on human factors in computing systems, 1993.

[68] Zhang P and Soergel D. Towards a comprehensive model of the cognitive process and mechanisms of individual sensemaking. Journal of American Society of Information Science and Technology 2014; 65(9): 1733-1756.

[69] Hansen P. User interface design for IR interaction: A task-oriented approach. In: Proceedings of the third international conference on the conceptions of the library and information science, 1999, pp. 191-205.

[70] O'Day V and Jeffries R. Orienteering in an information landscape: How information seekers get from here to there. In: Proceedings of the INTERCHI conference on human factors in computing systems (CHI'93), 1993, pp. 438-445.

[71] Marchionini G, Geisler G and Brunk B. Agileviews: A human-centered framework for interfaces to information spaces. In: Proceedings of the American Society for Information Science, 2000, pp. 271-280.

[72] Hansen P and Järvelin K. Effects of foreign language and task scenario on relevance assessment. Journal of Documentation 2005; 61(5): 623-639.

[73] Hearst M. Search user interfaces. New York: Cambridge University Press, 2009. 
[74] Zhang M and Quintana C. Scaffolding strategies for supporting middle school students' online inquiry processes. Computers and Education 2011; 58: 181-196.

[75] Kuhlthau CC, Maniotes LK and Caspari AK. Guided inquiry: Learning in the 21st century. Westport, CT: Libraries Unlimited, 2007.

[76] Kuhlthau CC, Maniotes LK and Caspari AK. Guided inquiry design: A framework for inquiry in your school. Santa Barbara, CA: Libraries Unlimited, 2012.

[77] Quintana C, Reiser BJ, Davis EA et al. A scaffolding design framework for software to support science inquiry. Journal of the Learning Sciences 2004; 13: 337-386.

[78] White RW and Huang J. Assessing the scenic route: Measuring the value of search trails in web logs. In: Proceedings of the $33 r d$ annual international ACM SIGIR conference on research and development in information retrieval, 2010, pp. 587-594.

[79] Collins-Thompson K, Bennett PN, White RW et al. Personalizing web search results by reading level. In: Proceedings of the twentieth ACM international conference on information and knowledge management (CIKM), 2011, pp. 403-412.

[80] Tan C, Gabrilovich E and Pang B. To each his own: Personalized content selection based on text comprehensibility. In: Proceedings of the fifth ACM international conference on web search and data mining (WSDM), 2012, pp. $233-242$.

[81] Eickhoff C, Dungs S and Tran V. An eye-tracking study of query reformulation. In: Proceedings of the 38th annual international ACM SIGIR conference on research and development in information retrieval, 2015, pp. 13-22.

[82] Buckland M. Information and information systems. New York: Praeger, 1991. 